\title{
Health-related quality of life and its predictors among adults living with HIV/AIDS and receiving antiretroviral therapy in Pakistan
}

\author{
Ali Ahmed ${ }^{1} \cdot$ Muhammad Saqlain $^{2} \cdot$ Naila Bashir $^{3} \cdot$ Juman Dujaili $^{1} \cdot$ Furqan Hashmi $^{4} \cdot$ Faizan Mazhar $^{5} \cdot$ Amjad Khan $^{6}$. \\ Musarat Jabeen $^{7} \cdot$ Ali Blebil $^{1} \cdot$ Ahmed Awaisu $^{8}$ iD
}

Accepted: 15 January 2021 / Published online: 13 February 2021

(C) The Author(s) 2021

\begin{abstract}
Background Health-related quality of life (HRQoL) is considered to be the fourth 90 of UNAIDS 90-90-90 target to monitor the effects of combination antiretroviral therapy (ART). ART has significantly increased the life expectancy of people living with HIV/AIDS (PLWHA). However, the impact of chronic infection on HRQoL remains unclear, while factors influencing the HRQoL may vary from one country to another. The current study aimed to assess HRQoL and its associated factors among PLWHA receiving ART in Pakistan.

Methods A cross-sectional descriptive study was conducted among PLWHA attending an ART centre of a tertiary care hospital in Islamabad, Pakistan. HRQoL was assessed using a validated Urdu version of EuroQol 5 dimensions 3 level (EQ5D-3L) and its Visual Analogue Scale (EQ-VAS).

Results Of the 602 patients included in the analyses, 59.5\% $(n=358)$ reported no impairment in self-care, while $63.1 \%$ $(n=380)$ were extremely anxious/depressed. The overall mean EQ-5D utility score and visual analogue scale (EQ-VAS) score were 0.388 (SD: 0.41) and 66.20 (SD: 17.22), respectively. Multivariate linear regression analysis revealed that the factors significantly associated with HRQoL were: female gender; age > 50 years; having primary and secondary education; > 1 year since HIV diagnosis; HIV serostatus AIDS-converted; higher CD 4 T lymphocytes count; detectable viral load; and increased time to ART.

Conclusions The current findings have shown that PLWHA in Pakistan adherent to ART had a good overall HRQoL, though with significantly higher depression. Some of the factors identified are amenable to institution-based interventions while mitigating depression to enhance the HRQoL of PLWHA in Pakistan. The HRQoL determined in this study could be useful for future economic evaluation studies for ART and in designing future interventions.
\end{abstract}

Keywords Pakistan $\cdot$ Health-related quality of life $\cdot$ HIV/AIDS $\cdot$ EQ-5D-3L $\cdot$ Antiretroviral therapy $\cdot$ Predictors

\section{Background}

Low and middle-income countries (LMIC) like Pakistan experience relatively high rates of new human immunodeficiency virus (HIV) infections $[1,2]$. Pakistan's progress towards The Joint United Nations Programme on HIV/ AIDS (UNAIDS)' 90-90-90 goal is lagging behind as only $14 \%$ of people living with human immunodeficiency virus and acquired immunodeficiency syndrome (HIV/AIDS) (PLWHA) are aware of their HIV status, only $10 \%$ are on treatment, while data on viral suppression are unavailable

Ahmed Awaisu

aawaisu@qu.edu.qa

Extended author information available on the last page of the article
[3]. Since the advent of safe and effective antiretroviral therapy (ART), AIDS has transformed from a terminal disease to a manageable chronic disease $[4,5]$. The use of Highly Active Antiretroviral Therapy (HAART) in PLWHA results in viral load (VL) suppression [6], boosts the immune system [7], and contributes to improving their health-related quality of life (HRQoL) [8]. A new "beyond viral suppression" model proposed to add a "fourth 90" to the UNAIDS" 90-90-90 target, is to ensure that $90 \%$ of PLWHA with VL suppression have good HRQoL [9, 10].

HRQoL is a multidimensional concept [11] that includes the level of independence, physical health, spiritual health, social health, psychological health, and environmental health [12]. The relationship between HIV/AIDS, ART and HRQoL is a complex one [13]. ART helps to prevent 
the spread of the disease and results in improved QoL and the well-being of PLWHA, but the continuous use of ART required to sustain viral suppression can lead to adverse effects such as lipodystrophy, diarrhoea, and other drugrelated symptoms, which have also been shown to affect HRQoL [14, 15]. Several other factors such as age [16], gender [17], socioeconomic factors [8], VL [10, 18], CD4 T lymphocytes count [19], education [8], depression [20, 21], comorbidities [22], stigma and discrimination [23], mental health [24], behavioural factors [22] and poor adherence to ART [25], have been reported to influence HRQoL in PLWHA. However, the factors influencing HRQoL among PLWHA may vary from one setting or country to another and the development of interventions to specific patients' population should take these differences into consideration.

HRQoL is an important measure that enables healthcare workers to understand patients' perceived satisfaction and perception of illness [26]. Its assessment helps to monitor interventions and develop a variety of health strategies to improve the overall health of the community [11, 27]. In particular, HRQoL of PLWHA receiving ART is essential for monitoring the impact of drug therapy on the progression of the disease [28, 29]. To the best of our knowledge, there was no study conducted to assess HRQoL and the factors influencing it among PLWHA in Pakistan. To date, no HRQoL study has been conducted in Pakistan and therefore a baseline study is deemed necessary to inform health authorities in designing evidence-based interventions that are culturally-sensitive and customized to PLWHA in Pakistan. Generic tools such as the EuroQol five dimension (EQ5D) scale, World Health Organization Quality of Life (WHOQOL) instrument, and 36-Item Short Form Health Survey (SF-36) questionnaire, as well as disease-specific tools such as Medical Outcomes Study HIV Health Survey (MOS-HIV), short version of WHOQOL-HIV (WHOQOL HIV Bref) have been used to measure HRQoL in HIV patients $[11,29]$. In the present study, the generic EQ-5D three-level (EQ-5D-3L) questionnaire was used to permit a comparison of HRQoL across different disease conditions and sub-populations [30]. Another advantage of the EQ-5D is that it generates health utility scores that can be used in economic evaluation studies as well $[8,31]$. Thus, this study aimed to: (1) evaluate HRQoL in PLWHA receiving ART; (2) determine the sensitivity of the EuroQol questionnaire to differentiate the HRQoL in different stages of HIV infection, CD4 T lymphocytes, and VL and; (3) identify factors influencing HRQoL in Pakistani patients with diverse cultural background.

\section{Method}

\section{Study design and setting}

This was a cross-sectional study using a validated generic HRQoL questionnaire. The study was conducted from July 2019 to September 2019 at the ART Centre in PIMS Hospital, a 947-bed hospital located in Islamabad Capital Territory (ICT), Pakistan. The ART Centre at PIMS is the largest centre of HIV referral cases in Pakistan, serving more than 4200 registered PLWHA who receive free-of-charge HIV treatment. Approximately 20 to 25 patients visit the ART centre daily for their medical needs. The centre was selected because of its geographical location and its capacity in serving a large number of patients from different ethnic backgrounds and various regions of Pakistan.

\section{Participants' characteristics}

Patients were included in the study if they fulfilled the following criteria: aged 18 years or older, diagnosed with HIV for $\geq 6$ months, have a recent CD- 4 T cells count and viral load tests (no more than four weeks at the time of the interview), and can understand and converse in Urdu language (the national language of Pakistan). Only PLWHA who were taking ART on a regular basis were included in the study. Adherence to ART was confirmed by checking patients' hospital pharmacy refill records. Prescription medical claims records can be used to estimate medication adherence based on the refill rate. Patients may routinely be defined as 'adherent patients' if the amount of medication given is at least $80 \%$ based on the medication possession ratio (MPR), which is defined as the day-to-day delivery of the medication divided by the number of days the patient should take the medication [32]. Patients with terminal illness, visual impairment, hearing impairment, non-adherence to ART, multiple comorbidities, cognitive impairment, and those unable to complete the surveys were excluded from the study.

\section{Sample size and sampling technique}

A simple random sampling technique was used to collect data from PLWHA who fulfilled the eligibility criteria outlined above. Every PLWHA registered at the ART Centre who fulfilled the inclusion criteria had equal chance of being enrolled into the study. The sample size of 599 was calculated using the Raosoft calculator by taking a total of 180,000 HIV patients, $95 \%$ confidence interval and $4 \%$ margin of error [33]. The sample size was adjusted for unintentional error/missing rate [34] using the following formula. 
$n=\frac{n}{(1-d)}$

where, $n=599$, and $\mathrm{d}=5 \%$. The formula provided a required sample size of 628.95 patients $\left(n=599+\mathrm{n}_{1}=29.95\right)$. Overall, $630 \mathrm{HIV}$ patients were approached. Out of these, 610 patients voluntarily consented to participate in the study. Six hundred and two patients were included in the final analysis.

\section{Study instrument and data collection procedure}

EuroQol quality of life scale EQ-5D-3L and Visual Analogue Scale (VAS) have been widely used for HRQoL assessment [35]. The study protocol was registered in EuroQol Research Foundation (ID 29767) and the psychometrically validated Urdu version of EQ-5D-3L tool was obtained. EQ-5D assesses five health domains: mobility, self-care, usual activities, discomfort/pain, anxiety/depression, and each dimension is evaluated at three levels (no problem, some or moderate problem, and extreme problem). The health status of the study population can be described as 243 different health states. These health states produce a value set (index score) ranging from 1 (for the best health state, 11111) to -0.594 (for the worst health state, 33333), where a negative sign indicates QoL worse than death. EQ5D-3L also includes a visual analogue scale (EQ-VAS), which is a vertical $20-\mathrm{cm}$ calibrated line ranging from 0 to 100. The 0 describes feeble health and 100 represents the best health. The EQ-5D-3L and EQ-VAS were previously validated in Pakistan and have been used to evaluate HRQoL in patients with tuberculosis [36], hepatitis B [37], diabetes [38], and hypertension [27, 39]. The internal consistency reliability of the questionnaire (Cronbach alpha 0.72) were ensured in our study.

The data collection was carried out for a period of three months (from July 2019 to September 2019). Each person living with HIV/AIDS attending the ART centre, was approached and invited to participate in the study by the head nurse (M.J.) and HIV physician (N.B.). Those who agreed to participate were informed about the nature and purpose of the study. Each consented patient was assessed against the pre-defined inclusion and exclusion criteria by the principal investigator (A.A.). Data collection was performed at the study setting (ART Centre in PIMS Hospital). The data collection consisted of two parts: the first part covered the socio-demographic and clinical characteristics of the participants, while the second part consisted of the EQ-5D-3L and EQ-VAS. Eligible participants were asked to complete their socio-demographic characteristics (gender, age, educational level, employment status, relationship status) in Part 1 of the data collection instrument, which were confirmed from their medical records. They were also requested to self-assess their HRQoL using EQ-5D-3L and
EQ-VAS in part 2 of the data collection instrument. Participants who were unable to understand any part of the questionnaire or were unable to self-administer the survey were assisted by the investigator collecting the data. The participants were instructed to read the brief instruction on the top of the page. Participants' correct understanding was ensured, and they were encouraged to complete every question in chronological order without skipping any items. The total completion time was approximately 15-25 min. Participants' clinical parameters, including CD4 lymphocyte count, HIV serostatus, and VL, were retrieved from the patients' medical records. No monetary benefits or other incentives were given to study participants due to lack of funding for the study.

\section{Statistical analysis}

Data were coded in MS Excel 2016 and imported into Statistical Package for Social Sciences (SPSS) version 21 (IBM SPSS $®$ Statistics for Windows, version 26.0; IBM Corp, Armonk, NY, USA) for analysis. Descriptive and inferential statistics were applied as appropriate and depending on the nature of the data. Numerical variables were expressed as means and standard deviations, while categorical variables were presented as frequencies and percentages. HRQoL domains were classified as no problem, some problem and severe problem. The HRQoL utility scores (index values) were calculated using the original 1995 UK population data due to lack of utility score data from the Pakistan population [40]. Independent sample t test and One-Way ANOVA test were applied to determine the differences in HRQoL (EQ-5D index score and EQ-VAS score) by patient characteristics. Only variables responsible for significant variation $(p<0.05)$ in HRQoL in the univariate analyses were included in the regression analysis. A multivariate linear regression model was applied to find significant predictors of HRQoL among the PLWHA. Before regression analysis normal distribution of data was assessed using Shapiro-wilk test. Multivariable linear regression models with the same variables of the full and final models were ran only to assess multicollinearity using variance inflation factor diagnostics (VIF). The results are presented as unstandardized beta coefficients accompanied by $95 \%$ confidence intervals (CIs). A $p$-value of $<0.05$ was considered statistically significant.

\section{Ethical considerations}

The ethical approval for the study was obtained from the Ethical Review Board (ERB) of ART Centre, Pakistan Institute of Medical Sciences (PIMS) and National AIDS Control Programme (NACP), Pakistan (Approval No; 1827). The objectives and procedures of the study were explained to the patients verbally. Informed consent was obtained from 
PLWHA who agreed to participate in the study. Moreover, potential participants were informed that participation in the study was voluntary and that they could withdraw at any time. All information collected would be kept confidential and was available to the research team only. All study procedures adopted complied with the principles of the Declaration of Helsinki, Good Clinical Practice and within the applicable laws and regulations of research involving human subjects in Pakistan.

\section{Results}

\section{Sociodemographic and clinical characteristics of PLHWA in Pakistan}

Of the 602 patients included in the final analysis, $65.3 \%$ $(n=393)$ were male, $41 \%(n=247)$ belonged to the age group $26-50$ years, and $37.7 \%(n=227)$ had primary school education. Furthermore, about half $(48.5 \%, n=292)$ of the participants were symptomatic, $38.9 \%(n=234)$ had CD $4 \mathrm{~T}$ cells in the range of $200-500$ cells $/ \mathrm{mm}^{3}$, more than half $(51.7 \%, n=311)$ had non-detectable viral load, and $64.6 \%(n=389)$ got infection by injecting drugs (Table 1$)$.

\section{EQ-5D-3L scores and health states of PLHWA in Pakistan}

The overall mean EQ-5D utility score was 0.388 (SD: $0.41)$. Of the 602 participants, more than half $56.0 \%$, $(n=337)$ reported no impairment in mobility, $59.5 \%$ $(n=358)$ reported no impairment in self-care, while $53.5 \%$ $(n=322)$ stated no problems in usual activities. Nevertheless, $50.3 \%(n=303)$ of the patients reported moderate pain/discomfort and $63.1 \%(n=380)$ patients were extremely anxious/depressed. The overall mean EQ-5D $3 \mathrm{~L}$ visual analogue scale (EQ-VAS) score was 66.20 (SD: 17.22) (Table 2).

Overall, 24 different health states were observed across the entire sample. Sixty-eight $(11.3 \%)$ of the respondents reported a complete healthy state (state: 11111 , index value: 1.00$)$ with no impairment in any domain, $86(14.3 \%)$ indicated a moderate problem in the first four domains and severe impairment in the fifth domain (state: 22223, index value: 0.082$)$, while $23(3.8 \%)$ reported worst health state (state: 33333 , index value: -0.594$)$ with extreme impairment in all the five domains of EQ-5D-3L (Table 3).
Table 1 Sociodemographic and clinical characteristics of PLWHA in Pakistan $(n=602)$

\begin{tabular}{|c|c|}
\hline Variable & $n(\%)$ \\
\hline \multicolumn{2}{|l|}{ Gender } \\
\hline Male & $393(65.3)$ \\
\hline Female & $189(31.4)$ \\
\hline Transgender & $20(3.3)$ \\
\hline \multicolumn{2}{|l|}{ Age (years) } \\
\hline $18-25$ & $157(26.1)$ \\
\hline $26-50$ & $247(41.0)$ \\
\hline$>50$ & $198(32.9)$ \\
\hline \multicolumn{2}{|l|}{ Marital status } \\
\hline Single & $227(37.7)$ \\
\hline Married/In relationship & $158(26.2)$ \\
\hline Divorced/separated & $163(27.1)$ \\
\hline Widowed & $54(9.0)$ \\
\hline \multicolumn{2}{|l|}{ Level of education } \\
\hline Illiterate & $173(28.7)$ \\
\hline Primary & $227(37.7)$ \\
\hline Secondary & $164(27.2)$ \\
\hline Tertiary & $38(6.3)$ \\
\hline \multicolumn{2}{|l|}{ Employment status } \\
\hline Employed & $209(34.7)$ \\
\hline Unemployed & $393(65.3)$ \\
\hline \multicolumn{2}{|c|}{ Duration since HIV diagnosis (years) } \\
\hline$<1$ & $181(30.1)$ \\
\hline $1-5$ & $237(39.4)$ \\
\hline $6-10$ & $120(19.9)$ \\
\hline$>10$ & $64(10.6)$ \\
\hline \multicolumn{2}{|l|}{ HIV serostatus } \\
\hline Asymptomatic & $290(48.2)$ \\
\hline Symptomatic & $292(48.5)$ \\
\hline AIDS-converted & $20(3.3)$ \\
\hline \multicolumn{2}{|l|}{ CD4 count } \\
\hline$<200$ cells $/ \mathrm{mm}^{3}$ & $190(31.6)$ \\
\hline $200-500$ cells $/ \mathrm{mm}^{3}$ & $234(38.9)$ \\
\hline$>500$ cells $/ \mathrm{mm}^{3}$ & $178(29.6)$ \\
\hline \multicolumn{2}{|l|}{ Viral load } \\
\hline Detectable & $291(48.3)$ \\
\hline Non-detectable & $311(51.7)$ \\
\hline \multicolumn{2}{|l|}{ Time on ART } \\
\hline$<12$ months & $225(37.4)$ \\
\hline $12-48$ months & $140(23.3)$ \\
\hline$>48$ months & $237(39.4)$ \\
\hline \multicolumn{2}{|c|}{ How you get infected with HIV? } \\
\hline No idea & $10(1.7)$ \\
\hline Blood products & $120(19.9)$ \\
\hline Injecting drugs & $389(64.6)$ \\
\hline Sex with men & $50(8.3)$ \\
\hline Sex with women & $33(5.5)$ \\
\hline
\end{tabular}


Table 2 EQ-5D-3L scores of PLHWA in Pakistan $(n=602)$

\begin{tabular}{lc}
\hline Variable & Mean (SD) \\
\hline EQ-5D index score [Mean (SD)] & $0.388(0.41)$ \\
VAS score [Mean (SD)] & $66.20(17.22)$ \\
Variable & $n(\%)$ \\
Mobility & \\
No problem & $337(56.0)$ \\
Some problem & $201(33.4)$ \\
Confined to bed & $43(7.1)$ \\
Self-care & \\
No problem & $358(59.5)$ \\
Some problem & $201(33.4)$ \\
Unable to self-care & $43(7.1)$ \\
Usual activities & \\
No problem & $322(53.5)$ \\
Some problem & $247(41.0)$ \\
Unable to perform activities & $33(5.5)$ \\
Pain/discomfort & \\
No pain & $256(42.5)$ \\
Moderate pain & $303(50.3)$ \\
Extreme pain & $43(7.1)$ \\
Depression/anxiety & \\
Not anxious/depressed & \\
Moderate anxious/depressed & $141(23.4)$ \\
Extremely anxious/depressed & $380(63.1)$ \\
\hline &
\end{tabular}

\section{Difference in HRQoL by patients' characteristics}

Independent sample t-test revealed that EQ-5D scores significantly differed by employment status $(P<0.001)$. One-way ANOVA test revealed that EQ-5D scores varied significantly by gender $(P<0.001)$, age $(P<0.001)$, marital status $(P<0.001)$, and level of education $(P<0.001)$. The findings indicated that asymptomatic HIV patients had significantly higher mean EQ-5D score $(0.58 \pm 0.37)$ than symptomatic HIV patients $(0.26 \pm 0.34)$ and AIDSconverted patients $(-0.38 \pm 0.22)$. In addition, HRQoL score significantly differed according to the participants' HIV serostatus $(P<0.001)$, CD4 count $(P<0.001)$, viral load $(P<0.001)$, time to ART $(P<0.001)$, and the route of HIV infection $(P<0.001)$ (Table 4$)$.

Similarly, VAS scores significantly differed by employment status $(P<0.001)$, gender $(P<0.001)$, age $(P<0.001)$, marital status $(P<0.001)$, and level of education $(P<0.001)$. Furthermore, asymptomatic HIV patients had the highest mean VAS score $(74.75 \pm 15.27)$ compared to symptomatic HIV patients $(59.15 \pm 15.02)$ and AIDS-converted patients $(45.10 \pm 5.12)$. Additionally, patients with CD4 count $>500$ cells $/ \mathrm{mm}^{3}$ had significantly higher VAS score $(86.46 \pm 8.25)$ than patients with CD4 count 200-500
Table 3 Health states of PLHWA in Pakistan $(n=602)$

\begin{tabular}{|c|c|c|c|}
\hline Serial No & EQ-5D states & $n(\%)$ & EQ-5D index value \\
\hline 1 & 11111 & $68(11.3)$ & 1.000 \\
\hline 2 & 11112 & $80(13.3)$ & 0.848 \\
\hline 3 & 11113 & $23(3.8)$ & 0.414 \\
\hline 4 & 11121 & $13(2.2)$ & 0.796 \\
\hline 5 & 11122 & $37(6.1)$ & 0.725 \\
\hline 6 & 11123 & $14(2.3)$ & 0.255 \\
\hline 7 & 11212 & $7(1.2)$ & 0.812 \\
\hline 8 & 11213 & $20(3.3)$ & 0.378 \\
\hline 9 & 11222 & $17(2.8)$ & 0.689 \\
\hline 10 & 11223 & $13(2.2)$ & 0.255 \\
\hline 11 & 12113 & $17(2.8)$ & 0.310 \\
\hline 12 & 12123 & $7(1.2)$ & 0.187 \\
\hline 13 & 12213 & $21(3.5)$ & 0.274 \\
\hline 14 & 21123 & $23(3.8)$ & 0.222 \\
\hline 15 & 21213 & $13(2.2)$ & 0.309 \\
\hline 16 & 21223 & $30(5.0)$ & 0.186 \\
\hline 17 & 22113 & $7(1.2)$ & 0.241 \\
\hline 18 & 22123 & $33(5.5)$ & 0.118 \\
\hline 19 & 22223 & $86(14.3)$ & 0.082 \\
\hline 20 & 22233 & $20(3.3)$ & -0.181 \\
\hline 21 & 23223 & $10(1.7)$ & -0.028 \\
\hline 22 & 23323 & $10(1.7)$ & -0.086 \\
\hline 23 & 32223 & $10(1.7)$ & -0.163 \\
\hline \multirow[t]{2}{*}{24} & 33333 & $23(3.8)$ & -0.594 \\
\hline & Total & $602(100.0)$ & \\
\hline
\end{tabular}

cells $/ \mathrm{mm}^{3}(62.13 \pm 12.06)$ and CD 4 count $<200$ cells $/ \mathrm{mm}^{3}$ $(52.21 \pm 9.83)$. VAS scores also significantly differed by HIV serostatus $(P<0.001)$, viral load $(P<0.001)$, time to ART $(P<0.001)$, and route of HIV infection $(P<0.001)$ (Table 4).

\section{Regression analysis}

Standard multiple regression analysis was used to assess the ability of socio-demographic and clinical characteristics to predict HRQoL in PLWHA in Pakistan as indicated by EQ-5D index (Table 5). Preliminary analyses were conducted to ensure no violation of the assumptions of normality, linearity, multicollinearity and homoscedasticity. A good fit model for multivariate linear regression was determined $(F=178.82, P<0.001$, adjusted $\left.\mathrm{R}^{2}=0.881\right)$. The regression model results (unstandardized $\beta ; p$-value) revealed that factors significantly associated with HRQoL were: female vs. male $(0.118 ;<0.001)$; age $>50$ years vs. $18-25$ years $(0.054$; $0.042)$; primary education vs. illiterate $(-0.139 ;<0.001)$, secondary education vs. illiterate $(-0.163 ;<0.001)$; non 
Table 4 Difference in EQ-5D scores by sample characteristics $(n=602)$

\begin{tabular}{|c|c|c|c|c|c|c|}
\hline Characteristics & $\begin{array}{l}\text { EQ-5D-3L } \\
\text { Mean } \pm S D\end{array}$ & $95 \% \mathrm{CI}$ & $P$-value & $\begin{array}{l}\text { EQ-VAS } \\
\text { Mean } \pm S D\end{array}$ & $95 \% \mathrm{CI}$ & $P$-value \\
\hline Gender $^{\mathrm{b}}$ & & & $<0.001$ & & & $<0.001$ \\
\hline Male & $0.41 \pm 0.44$ & $0.37-0.45$ & & $68.90 \pm 16.78$ & $67.24-70.57$ & \\
\hline Female & $0.29 \pm 0.32$ & $0.25-0.35$ & & $58.73 \pm 15.99$ & $56.43-61.02$ & \\
\hline Trans gender & $0.81 \pm 0.03$ & $0.80-0.83$ & & $83.50 \pm 4.89$ & $81.20-85.79$ & \\
\hline $\operatorname{Age}^{b}$ & & & $<0.001$ & & & $<0.001$ \\
\hline $18-25$ years & $0.63 \pm 0.32$ & $0.58-0.68$ & & $78.40 \pm 14.38$ & $76.13-80.67$ & \\
\hline $26-50$ years & $0.52 \pm 0.33$ & $0.48-0.56$ & & $67.61 \pm 17.11$ & $65.46-69.75$ & \\
\hline$>50$ years & $0.03 \pm 0.32$ & $-0.01-0.07$ & & $54.74 \pm 11.06$ & $53.19-56.29$ & \\
\hline Marital status $^{\mathrm{b}}$ & & & $<0.001$ & & & $<0.001$ \\
\hline Single & $0.57 \pm 0.39$ & $0.52-0.62$ & & $75.06 \pm 14.81$ & $73.19-76.93$ & \\
\hline Married/relationship & $0.39 \pm 0.35$ & $0.33-0.44$ & & $64.30 \pm 17.71$ & $61.52-67.08$ & \\
\hline Divorced/separated & $0.20 \pm 0.38$ & $0.14-0.26$ & & $59.14 \pm 15.49$ & $56.74-61.53$ & \\
\hline Widowed & $0.19 \pm 0.39$ & $0.09-0.30$ & & $55.74 \pm 14.74$ & $51.71-59.76$ & \\
\hline Level of education ${ }^{\mathrm{b}}$ & & & $<0.001$ & & & $<0.001$ \\
\hline Illiterate & $0.29 \pm 0.51$ & $0.21-0.36$ & & $62.25 \pm 16.91$ & $59.71-64.79$ & \\
\hline Primary & $0.36 \pm 0.33$ & $0.32-0.40$ & & $61.89 \pm 15.49$ & $59.86-63.92$ & \\
\hline Secondary & $0.39 \pm 0.32$ & $0.34-0.44$ & & $69.14 \pm 13.44$ & $67.07-71.22$ & \\
\hline Tertiary & $0.48 \pm 0.29$ & $0.41-0.55$ & & $97.15 \pm 4.59$ & $95.59-98.61$ & \\
\hline Employment status $^{\mathrm{a}}$ & & & $<0.001$ & & & $<0.001$ \\
\hline Employed & $0.55 \pm 0.37$ & $0.49-0.59$ & & $72.34 \pm 16.69$ & $70.06-74.62$ & \\
\hline Non-employed & $0.30 \pm 0.41$ & $0.26-0.34$ & & $62.92 \pm 16.62$ & $61.27-64.57$ & \\
\hline Since HIV diagnosed (years) ${ }^{b}$ & & & $<0.001$ & & & $<0.001$ \\
\hline$<1$ & $0.57 \pm 0.39$ & $0.52-0.62$ & & $74.47 \pm 15.99$ & $72.12-76.82$ & \\
\hline $1-5$ & $0.39 \pm 0.35$ & $0.33-0.43$ & & $62.91 \pm 15.90$ & $60.87-69.94$ & \\
\hline $6-10$ & $0.20 \pm 0.38$ & $0.14-0.26$ & & $58.25 \pm 13.69$ & $55.77-60.72$ & \\
\hline$>10$ & $0.19 \pm 0.39$ & $0.08-0.31$ & & $69.84 \pm 20.62$ & $64.68-75.00$ & \\
\hline HIV serostatus ${ }^{b}$ & & & $<0.001$ & & & $<0.001$ \\
\hline Asymptomatic & $0.58 \pm 0.37$ & $0.53-0.62$ & & $74.75 \pm 15.27$ & $72.99-76.52$ & \\
\hline Symptomatic & $0.26 \pm 0.34$ & $0.22-0.29$ & & $59.15 \pm 15.02$ & $57.41-60.87$ & \\
\hline AIDS converted & $-0.38 \pm 0.22$ & $-0.48-0.28$ & & $45.10 \pm 5.12$ & $42.59-47.40$ & \\
\hline CD4 count ${ }^{\mathrm{b}}$ & & & $<0.001$ & & & $<0.001$ \\
\hline$<200$ & $0.03 \pm 0.30$ & $-0.09-0.07$ & & $52.21 \pm 9.83$ & $50.80-53.61$ & \\
\hline $200-500$ & $0.34 \pm 0.26$ & $0.31-0.37$ & & $62.13 \pm 12.06$ & $60.58-63.69$ & \\
\hline$>500$ & $0.83 \pm 0.22$ & $0.79-0.87$ & & $86.46 \pm 8.25$ & $85.23-87.68$ & \\
\hline Viral load $^{\mathrm{a}}$ & & & $<0.001$ & & & $<0.001$ \\
\hline Detectable & $0.22 \pm 0.34$ & $0.18-0.26$ & & $58.07 \pm 12.77$ & $56.60-59.54$ & \\
\hline Non-detectable & $0.55 \pm 0.41$ & $0.50-0.59$ & & $73.79 \pm 17.40$ & $71.85-75.73$ & \\
\hline Time on $\mathrm{ART}^{\mathrm{b}}$ & & & $<0.001$ & & & $<0.001$ \\
\hline$<12$ months & $0.50 \pm 0.35$ & $0.45-0.55$ & & $72.97 \pm 14.15$ & $71.11-74.83$ & \\
\hline $12-48$ months & $0.56 \pm 0.32$ & $0.51-0.62$ & & $66.64 \pm 18.09$ & $63.61-69.66$ & \\
\hline$>48$ months & $0.18 \pm 0.41$ & $0.13-0.23$ & & $59.49 \pm 16.84$ & $57.33-61.64$ & \\
\hline How you get infected with HIV? ${ }^{b}$ & & & $<0.001$ & & & $<0.001$ \\
\hline No idea & $0.12 \pm 0.17$ & $0.09-0.17$ & & $55.35 \pm 16.49$ & $51.15-59.55$ & \\
\hline Blood products & $0.30 \pm 0.46$ & $0.21-0.39$ & & $69.01 \pm 14.63$ & $66.35-71.64$ & \\
\hline Injecting drugs & $0.40 \pm 0.41$ & $0.3-0.44$ & & $66.45 \pm 16.42$ & $64.81-68.09$ & \\
\hline Sex with women & $0.34 \pm 0.31$ & $0.26-0.43$ & & $57.40 \pm 14.11$ & $53.38-61.41$ & \\
\hline Sex with men & $0.69 \pm 0.32$ & $0.58-0.81$ & & $68.18 \pm 32.15$ & $56.77-79.58$ & \\
\hline
\end{tabular}

Bold values show a statistically significant difference

$E Q$ EuroQoL, $S D$ Standard deviation, $C I$ Confidence interval

${ }^{a}$ Independent samples $t$-test

${ }^{\mathrm{b}}$ One-Way ANOVA was applied to calculate $p$-value 
Table 5 Univariate and multivariable linear regression analyses to determine the predictors of HRQoL in PLHWA in Pakistan $(n=602)$

\begin{tabular}{|c|c|c|c|c|c|c|}
\hline \multirow[t]{2}{*}{ Patient characteristics } & \multicolumn{2}{|c|}{$\begin{array}{l}\text { EQ-5D index value (Univariate linear } \\
\text { regression) }\end{array}$} & \multirow[t]{2}{*}{$P$-value } & \multicolumn{2}{|c|}{$\begin{array}{l}\text { EQ-5D index value } \\
\text { (Multivariate linear regression) }\end{array}$} & \multirow[t]{2}{*}{$P$-value } \\
\hline & Unstandardized $\beta$ & $95 \% \mathrm{CI}$ & & Unstandardized $\beta$ & $95 \% \mathrm{CI}$ & \\
\hline \multicolumn{7}{|l|}{ Gender } \\
\hline Female vs. male & -0.110 & -0.179 to -0.0405 & 0.002 & 0.118 & $0.089-0.149$ & $<0.001$ \\
\hline Transgender vs. male & 0.404 & $0.225-0.584$ & $<0.001$ & -0.054 & $-0.137-0.027$ & 0.193 \\
\hline \multicolumn{7}{|l|}{ Age } \\
\hline $26-50$ years vs. $18-25$ years & -0.112 & -0.1763 to -0.0481 & 0.001 & 0.158 & $0.114-0.202$ & $<0.001$ \\
\hline$>50$ years vs. $18-25$ years & -0.606 & -0.673 to -0.539 & $<0.001$ & 0.054 & $0.002-0.106$ & 0.042 \\
\hline \multicolumn{7}{|l|}{ Marital status } \\
\hline Married/In relationship vs. single & -0.186 & -0.263 to -0.109 & $<0.001$ & 0.139 & $0.089-0.189$ & $<0.001$ \\
\hline Divorced/separated vs. single & -0.370 & -0.447 to -0.294 & $<0.001$ & 0.006 & $-0.036-0.048$ & 0.771 \\
\hline Widowed vs. single & -0.377 & -0.490 to -0.265 & $<0.001$ & -0.003 & $-0.059-0.052$ & 0.914 \\
\hline \multicolumn{7}{|l|}{ Level of education } \\
\hline Primary vs. illiterate & 0.0698 & $-0.005-0.145$ & 0.066 & -0.139 & -0.183 to -0.094 & $<0.001$ \\
\hline Secondary vs. illiterate & 0.105 & $0.025-0.186$ & 0.010 & -0.163 & -0.211 to -0.115 & $<0.001$ \\
\hline Tertiary vs. illiterate & 0.711 & $0.578-0.844$ & $<0.001$ & -0.021 & $-0.904-0.048$ & 0.550 \\
\hline \multicolumn{7}{|l|}{ Employment status } \\
\hline Non-employed vs. employed & -0.242 & -0.308 to -0.176 & $<0.001$ & -0.070 & -0.104 to -0.036 & $<0.001$ \\
\hline \multicolumn{7}{|l|}{ HIV since diagnosis (years) } \\
\hline $1-5$ vs. $<1$ & -0.125 & -0.203 to -0.048 & 0.002 & 0.289 & $0.233-0.346$ & $<0.001$ \\
\hline $6-10$ vs. $<1$ & -0.274 & -0.367 to -0.182 & $<0.001$ & 0.464 & $0.380-0.548$ & $<0.001$ \\
\hline$>10$ vs. $<1$ & -0.123 & -0.237 to --0.009 & -0.034 & 0.615 & $0.526-0.705$ & $<0.001$ \\
\hline \multicolumn{7}{|l|}{ HIV serostatus } \\
\hline Symptomatic vs. asymptomatic & -0.319 & -0.377 to -0.262 & $<0.001$ & -0.145 & -0.176 to -0.111 & $<0.001$ \\
\hline AIDS-converted vs. asymptomatic & -0.953 & -1.113 to -0.794 & $<0.001$ & -0.638 & -0.731 to -0.545 & $<0.001$ \\
\hline \multicolumn{7}{|l|}{ CD4 count } \\
\hline $200-500$ vs. $<200$ & 0.306 & $0.256-0.356$ & $<0.001$ & 0.173 & $0.125-0.221$ & $<0.001$ \\
\hline$>500$ vs. $<200$ & 0.798 & $0.744-0.852$ & $<0.001$ & 0.670 & $0.604-0.739$ & $<0.001$ \\
\hline \multicolumn{7}{|l|}{ Viral load } \\
\hline Detectable vs. non-detectable & -0.334 & -0.393 to -0.273 & $<0.001$ & -0.038 & -0.074 to -0.002 & 0.041 \\
\hline \multicolumn{7}{|l|}{ Time on ART } \\
\hline $12-48$ months vs. $<12$ months & 0.062 & $-0.017-0.141$ & 0.123 & -0.338 & -0.393 to -0.281 & $<0.001$ \\
\hline$>48$ months vs. $<12$ months & -0.320 & $-0.388-0.252$ & $<0.001$ & -0.712 & -0.790 to -0.634 & 0.001 \\
\hline \multicolumn{7}{|l|}{ How you get infected with HIV? } \\
\hline No idea vs. sex with men & -0.577 & -0.862 to -0.293 & $<0.001$ & -0.202 & -0.323 to -0.079 & 0.001 \\
\hline Blood products vs. sex with men & -0.391 & -0.546 to -0.237 & $<0.001$ & -0.262 & -0.329 to -0.194 & $<0.001$ \\
\hline Injecting drugs vs. sex with men & -0.294 & -0.437 to -0.151 & $<0.001$ & -0.037 & $-0.09-0.260$ & 0.251 \\
\hline Sex with women vs. sex with men & -0.352 & -0.529 to -0.175 & $<0.001$ & 0.211 & $0.139-0.282$ & $<0.001$ \\
\hline
\end{tabular}

Normality of data was assessed by QQ-plot and Shapiro-wilk test $(P>0.05)$

Model statistics: F: $178.82 ; P<0.001 ; \mathrm{R}^{2}: 0.885$, Adjusted $\mathrm{R}^{2}: 0.881$

employed vs. employed $(-0.070 ;<0.001)$; years with HIV $1-5$ years vs. $<1$ year $(0.289 ;<0.001), 6-10$ years vs. $<1$ year $(0.464 ;<0.001),>10$ years vs. $<1$ year $(0.615$; <0.001); HIV serostatus symptomatic vs. asymptomatic $(-0.145 ;<0.001)$, AIDS-converted vs. asymptomatic $(-0.638 ;<0.001)$; CD4 count $200-500$ cells/ $\mathrm{mm}^{3}$ vs. $<200$ cells $/ \mathrm{mm}^{3}(0.173 ;<0.001),>500$ cells/ $\mathrm{mm}^{3}$ vs. $<200$ cells $/ \mathrm{mm}^{3}(0.670 ;<0.001)$; viral load detectable $>20$ copies/mL vs. non-detectable $(-0.038$; $0.041)$; time on ART $12-48$ months vs. $<12$ months $(-0.338,<0.001),>48$ months vs. $<12$ months $(-0.712$; $0.001)$; no idea vs. sex with a man $(-0.202 ; 0.001)$, blood products vs. sex with a man $(-0.262 ;<0.001)$. 


\section{Discussion}

This is the first study in Pakistan that assessed the HRQoL of PLWHA who actively sought testing and receiving ART treatment. Therefore, the findings cannot be generalized to all PLWHA in Pakistan, particularly those not receiving treatment or not adherent to ART. Given the increasing incidence of HIV [1] in recent years, such a study was needed to evaluate the QoL of PLWHA in Pakistan to further develop tailored interventions. Our findings suggested that several independent factors such as male gender, transgender, young age, being employed, having higher CD-4 lymphocytes count, undetectable viral load, higher education level, and being asymptomatic were significantly associated with higher HRQoL.

The mean EuroQol 5D-3L score for PLWHA was 0.388, which could not be compared to the general population health as no data were available for Pakistan. Studies conducted in Brazil [8] and South Africa [41] reported a mean score of 0.82 and 0.80 , which were higher scores than the present study. Likewise, our findings showed a score lower than the average score of 0.74 found in another study conducted in England. [30]. Regarding EQ-VAS score, our results are consistent with the South African study [41], but varied from Nglazi et al. [42] study that reported VAS median score of 90 for PLWHA adherent to ART. High level of depression is a significant predictor of lower HRQoL [43], with more than $63 \%$ of PLWHA having severe depression and anxiety in this study. Previous studies have shown that more than $10 \%$ of anxiety and depression, are key factors affecting HRQoL of PLWHA [44, 45]. Therefore, psychological interventions are needed in ART centres to improve HRQoL associated with mental health [46]. A randomized controlled trial conducted in Pakistan had reported that multi-component behavioural interventions had resulted in a significant reduction in anxiety and depression within 3 months in psychologically distressed adults [47].

In our study, older age and lower level of education were significant predictors of poor HRQoL; these results are in agreement with those obtained by Brazilian and Italian studies [8, 48]. Higher education levels may lead to a better capacity to cope with HIV, contribute to improvement of patient knowledge about the disease, and ultimately HRQoL domains $[49,50]$. HRQoL decreased with an increase in age, and these results are in agreement with those obtained by American, French and Brazilian studies [8, 15, 51]. Memory issues, anxiety and depression, gender, ethnicity, economic factors, and family situation have been identified as leading factors to lower HRQoL in individuals over 50 years of age with HIV [52, 53]. It is worthwhile to explore ageing in the presence of comorbidities, frailty, anxiety, and depression to improve survival and HRQoL in PLWHA $[8,54]$.
Male and transgender patients with HIV had better HRQoL compared to female patients based on ANOVA test, and these results are consistent with Zimbabwean and Italian studies $[48,55]$. In general, females are the most marginalized population, perhaps due to the high stigma and discrimination against them in Pakistan [56]. It can, therefore, be inferred that gender is a significant social determinant of health and that variations between gender-related HRQoL factors need to be addressed [57]. The current study found that the average HRQoL score of singles was more than married and widowed patients, which is consistent with the evidence from a previous study conducted in Ethiopia [58]. The finding that married or cohabiting people exhibiting a lower level of spiritual HRQoL may be attributed to couples' self-accusation of contracting and/or transferring the virus to one another or fear of losing their partner (due to AIDS-related causes) in the future [59]. However, some studies have reported that being in a relationship is a good predictor of quality life as it lowers the chances of loneliness and depression $[8,13,51]$. Unemployment was another predictor that resulted in lower HRQoL. This was also documented in other studies conducted in Vietnam and Japan $[60,61]$, because employment could be linked to higher social well-being of PLWHA. Regarding the sensitivity of EQ-5D, AIDS-converted patients demonstrated significantly lower HRQoL compared to symptomatic and asymptomatic patients, especially in physical health and pain, a finding that was also reported by other studies $[62,63]$. We enrolled only adherent patients and adherence is linked to viral suppression and subsequent asymptomatic state. However, we identified AIDS-converted and symptomatic patients, which may be attributed to several factors including drug interactions and viral drug resistance $[64,65]$, and potentially natural disease progression.

EQ-5D VAS showed a significant relationship between HRQoL domains and CD4 lymphocytes count of $>200$ cells $/ \mathrm{mm}^{3}$. With an increase in CD4 lymphocytes count, physical HRQoL improved. Similarly, other studies have reported CD4 lymphocytes count as an important predictor of other domains of QoL [11, 13, 66, 67]. Some studies have reported that lower CD4 count can affect mental health, possibly due to distress associated with infection progression $[68,69]$. Furthermore, the present study has reaffirmed that suppression of viral load is a good predictor of HRQoL. Likewise, several other studies have predicted viral suppression to enhance physical health $[18,51]$ and mental health [70], and this can only be accomplished through a consistent lifelong adherence to ART [71]. Another advantage of viral suppression is that it stops the transmission of HIV to a non-HIV partner, thus supporting the worldwide slogan of Undetectable $=$ Untransmittable [72]. 


\section{Strengths and limitations}

This is the first study to assess HRQoL in PLWHA in Pakistan with a large sample size. Second, EQ-5D VAS was used to support the results of EuroQol questionnaire in its sensitivity to differentiate QoL of PLWHA based on disease stage, VL and CD-4 T lymphocytes. Third, the current findings have implications for medical decision-making to design evidence-based interventions across age groups and cultures by considering the chronic nature of HIV infection and patient reported outcomes.

The study has some potential limitations that are inherent to most observational questionnaire-based studies. First, a cross-sectional study design was used in this study; therefore, reverse causality is possible, and the findings need to be confirmed using a longitudinal study design. Second, we have not enrolled patients who failed to follow up regularly based on their dispensing records; therefore, the results cannot be generalized to non-adherent HIV-AIDS patients. These results may only be generalized to the $10 \%$ patients who are actively seeking treatment from NACP. Finally, there is a potential for social desirability bias since the respondents are likely to underreport socially undesirable and behaviors and to over report more desirable attributes.

\section{Conclusion}

Overall, PLWHA in Pakistan who were adherent to their ART had demonstrated a good HRQoL. The EQ-5D-3L instrument was found to be sensitive in detecting QoL differences based on HIV clinical stages, number of CD-4 T lymphocytes and VL. Female gender, older age ( $>50$ years), low education, living with a partner/spouse, unemployed, being HIV symptomatic, AIDS-converted, lower CD 4 lymphocyte count, and detectable viral load were the factors that could predict poor HRQoL in our population and need special attention to improve HRQoL. Adherence to ART significantly improved HRQoL, except for psychological component, suggesting a need for training of healthcare professional caring for PLWHA on the screening and management of anxiety and depression among HIV patients. Psychoeducation should be conducted during initial evaluation to reduce negative beliefs regarding ART. The findings of the present study provide support for future work to design effective and holistic interventions that are culturally sensitive for Pakistan. Further studies on the determinants of health outcomes in this vulnerable population are also warranted.

Acknowledgements We acknowledge the National AIDS Control Program of Pakistan; the HIV case manager (Hassan Mansoor Malik) of PIMS Hospital for smooth conduct of study. We also acknowledge the PLWHA for voluntary participation in the study.
Author contributions A.A1 conceived and conceptualized the research idea. A.A1., M.S., N.B., J.D., F.K.H., F.M., A.K., M.J., A.B., and A.A2. designed and developed the study protocol. A.A1., N.B., M.J., and A.K. collected the data. M.S., A.A1., F.K.H, F.M., J.D., A.A2. analysed the data. M.S. and A.A1. generated the tables and wrote the first draft of the manuscript, which was subsequently reviewed by N.B., J.D., F.K.H., F.M., A.K., M.J., A.B., and A.A2. All authors approved the final version of the manuscript.

Funding Open access funding provided by the Qatar National Library.. We received no funding for this project.

Data availability Data sets used in this study can be requested from the corresponding author if justified.

\section{Compliance with ethical standards}

Conflict of interest The authors declare that they have no conflict of interest.

Ethical approval Ethical approval for the conduct of the study was obtained from the Ethical Review Board (ERB) of ART Centre, Pakistan Institute of Medical Sciences (PIMS) and National AIDS Control Programme, Pakistan (Approval \# 1827). All procedures performed in the study were in accordance with the good clinical practice and the Declaration of Helsinki 1964 and comparable ethical standards.

Consent to participate Informed consent was obtained from all the participants involved in this study.

Open Access This article is licensed under a Creative Commons Attribution 4.0 International License, which permits use, sharing, adaptation, distribution and reproduction in any medium or format, as long as you give appropriate credit to the original author(s) and the source, provide a link to the Creative Commons licence, and indicate if changes were made. The images or other third party material in this article are included in the article's Creative Commons licence, unless indicated otherwise in a credit line to the material. If material is not included in the article's Creative Commons licence and your intended use is not permitted by statutory regulation or exceeds the permitted use, you will need to obtain permission directly from the copyright holder. To view a copy of this licence, visit http://creativecommons.org/licenses/by/4.0/.

\section{References}

1. Ahmed, A., Hashmi, F. K., \& Khan, G. M. (2019). HIV outbreaks in Pakistan. The Lancet HIV, 6(7), e418.

2. Wahid, B. (2019). An update on the severe outbreak of HIV in Kot Imrana, Pakistan. The Lancet Infectious Diseases, 19(3), 241.

3. UNAIDS 2020 Pakistan data fact sheet. Available from https:// www.unaids.org/en/regionscountries/countries/pakistan. Accessed 12 May 2020.

4. Wada, N., et al. (2014). Cause-specific mortality among HIVinfected individuals, by CD4+ cell count at HAART initiation, compared with HIV-uninfected individuals. AIDS, 28(2), 257.

5. Kanters, S., et al. (2016). Comparative efficacy and safety of firstline antiretroviral therapy for the treatment of HIV infection: A systematic review and network meta-analysis. The lancet $H I V$, 3(11), e510-e520. 
6. Malta, M., et al. (2010). Adherence to antiretroviral therapy among HIV-infected drug users: A meta-analysis. AIDS and Behavior, 14(4), 731-747.

7. Ofotokun, I., et al. (2016). Antiretroviral therapy induces a rapid increase in bone resorption that is positively associated with the magnitude of immune reconstitution in HIV infection. AIDS, $30(3), 405$

8. Castro, R., et al. (2019). Health-related quality of life assessment among people living with HIV in Rio de Janeiro, Brazil: A crosssectional study. Quality of Life Research, 28(4), 1035-1045.

9. Lazarus, J. V., et al. (2016). Beyond viral suppression of HIV-the new quality of life frontier. BMC Medicine, 14(1), 94.

10. Cho, H., et al. (2019). The relationship between self-reported viral load suppression and quality of life among people living with HIV in South Carolina. AIDS Care, 32(9), 1-8.

11. Cooper, V., et al. (2017). Measuring quality of life among people living with HIV: A systematic review of reviews. Health and Quality of Life Outcomes, 15(1), 220.

12. Group, T.W. (1998). The World health organization quality of life assessment (WHOQOL): Development and general psychometric properties. Social Science and Medicine, 46(12), 1569-1585.

13. Emuren, L., et al. (2020). Predictors of health-related quality of life among military HIV-infected individuals. Quality of Life Research, 1, 1-15.

14. Dutra, B. S., et al. (2019). Changes health-related quality of life in HIV-infected patients following initiation of antiretroviral therapy: A longitudinal study. Brazilian Journal of Infectious Diseases, 23(4), 211-217.

15. Emuren, L., et al. (2017). Health-related quality of life among military HIV patients on antiretroviral therapy. PLoS ONE, 12(6), e178953.

16. Vincent, W., et al. (2017). HIV-related shame and health-related quality of life among older, HIV-positive adults. Journal of Behavioral Medicine, 40(3), 434-444.

17. Nobre, N., et al. (2017). Factors associated with the quality of life of people living with HIV in Finland. AIDS Care, 29(8), 1074-1078.

18. Call, S., et al. (2000). Health-related quality of life and virologic outcomes in an HIV clinic. Quality of Life Research, 9(9), 977-985.

19. Razera, F., Ferreira, J., \& Bonamigo, R. R. (2008). Factors associated with health-related quality-of-life in HIV-infected Brazilians. International Journal of STD and AIDS, 19(8), 519-523.

20. Ballester-Arnal, R., et al. (2016). A Spanish study on psychological predictors of quality of life in people with HIV. AIDS and Behavior, 20(2), 281-291.

21. Majeed, Z., et al. (2017). Depression partially mediated the relationship between basic psychological needs and quality of life among people living with HIV. AIDS Care, 29(11), 1399-1403.

22. Abebe Weldsilase, Y., et al. (2018). Health-related quality of life and associated factors among women on antiretroviral therapy in health facilities of Jimma Town, Southwest Ethiopia. Advances in Public Health, 2018, 1-12.

23. Zhou, G., et al. (2020). HIV symptom management self-efficacy mediates the relationship of internalized stigma and quality of life among people living with HIV in China. Journal of Health Psychology, 25(3), 311-321.

24. Gibson, K., et al. (2011). Mastery and coping moderate the negative effect of acute and chronic stressors on mental healthrelated quality of life in HIV. AIDS Patient Care and STDs, 25(6), 371-381.

25. Ahmed, A., et al. (2020). Concerns of HIV-positive migrant workers in COVID-19 pandemic: A call for action. Journal of Global Health, 10(2), 020342.
26. Group, W. (1998). Development of the World health organization WHOQOL-BREF quality of life assessment. Psychological Medicine, 28(3), 551-558.

27. Saleem, F., Hassali, M. A., \& Shafie, A. A. (2014). A crosssectional assessment of health-related quality of life (HRQoL) among hypertensive patients in Pakistan. Health Expectations, 17(3), 388-395.

28. Oguntibeju, O. O. (2012). Quality of life of people living with HIV and AIDS and antiretroviral therapy. HIV/AIDS, 4, 117.

29. Kall, M., et al. (2020). Patient-reported outcomes to enhance person-centred HIV care. The Lancet HIV, 7(1), e59-e68.

30. Miners, A., et al. (2014). Health-related quality-of-life of people with HIV in the era of combination antiretroviral treatment: A cross-sectional comparison with the general population. The Lancet HIV, 1(1), e32-40.

31. Santos, M., et al. (2016). Brazilian valuation of EQ-5D-3L health states: Results from a saturation study. Medical Decision Making, 36(2), 253-263.

32. Watanabe, J. H., Bounthavong, M., \& Chen, T. (2013). Revisiting the medication possession ratio threshold for adherence in lipid management. Current Medical Research and Opinion, 29(3), $175-180$.

33. Raosoft, I 2004 Sample size calculator by Raosoft, Inc. Available from: http://www.raosoft.com/samplesize.html. Accessed 10 June 2020.

34. Sakpal, T. (2010). Sample size estimation in clinical trial. Perspectives in Clinical Research, 1(2), 67-67.

35. Devlin, N. J., \& Brooks, R. (2017). EQ-5D and the EuroQol group: Past, present and future. Applied Health Economics and Health Policy, 15(2), 127-137.

36. Saleem, S., et al. (2018). Health-related quality of life among pulmonary tuberculosis patients in Pakistan. Quality of Life Research, 27(12), 3137-3143.

37. ul Haq, N., , et al. (2012). A cross sectional assessment of health related quality of life among patients with Hepatitis-B in Pakistan. Health and Quality of Life Outcomes, 10(1), 91.

38. Hassali, M. A., et al. (2016). A cross-sectional assessment of health-related quality of life among type 2 diabetic patients in Pakistan. Journal of Pharmacy and Bioallied Sciences, 8(1), 64.

39. Saqlain, M., et al. (2020). Potentially inappropriate medications use and its association with health-related quality of life among elderly cardiac patients. Quality of Life Research, 29(10), 2715-2724.

40. Dolan P., et al. (1995). A social tariff for EuroQol: results from a UK general population survey. Discussion Papers 138, Centre for Health Economics, University of York.

41. Louwagie, G. M., et al. (2007). Highly active antiretroviral treatment and health related quality of life in South African adults with human immunodeficiency virus infection: A cross-sectional analytical study. BMC Public Health, 7(1), 244.

42. Nglazi, M. D., et al. (2014). Quality of life in individuals living with HIV/AIDS attending a public sector antiretroviral service in Cape Town, South Africa. BMC Public Health, 14(1), 676.

43. Deshmukh, N. N., Borkar, A. M., \& Deshmukh, J. S. (2017). Depression and its associated factors among people living with HIV/AIDS: Can it affect their quality of life? Journal of Family Medicine and Primary Care, 6(3), 549.

44. Yang, Y., Thai, S., \& Choi, J. (2016). An evaluation of quality of life among Cambodian adults living with HIV/AIDS and using antiretroviral therapy: A short report. AIDS Care, 28(12), $1546-1550$.

45. Gibbie, T., et al. (2007). Depression, social support and adherence to highly active antiretroviral therapy in people living with HIV/ AIDS. Sexual Health, 4(4), 227-232. 
46. Freeman, M. C., et al. (2005). Integrating mental health in global initiatives for HIV/AIDS. The British Journal of Psychiatry, 187(1), 1-3.

47. Rahman, A., et al. (2016). Effect of a multicomponent behavioral intervention in adults impaired by psychological distress in a conflict-affected area of Pakistan: A randomized clinical trial. JAMA, 316(24), 2609-2617.

48. Venturini, A., et al. (2017). Quality of life in an Italian cohort of people living with HIV in the era of combined antiretroviral therapy (Evidence from IANUA study-investigation on antiretroviral therapy). AIDS Care, 29(11), 1373-1377.

49. Nobre, N., et al. (2018). HIV-related self-stigma and health-related quality of life of people living with HIV in Finland. Journal of the Association of Nurses in AIDS Care, 29(2), 254-265.

50. Rzeszutek, M., Gruszczyńska, E., \& Firląg-Burkacka, E. (2017). Coping profiles and subjective well-being among people living with HIV: Less intensive coping corresponds with better wellbeing. Quality of Life Research, 26(10), 2805-2814.

51. Préau, M., et al. (2007). Health-related quality of life in French people living with HIV in 2003: Results from the national ANRSEN12-VESPA study. AIDS, 21(Supp 1), S19-S27.

52. Jia, H., et al. (2004). Health-related quality of life among men with HIV infection: Effects of social support, coping, and depression. AIDS Patient Care and STDs, 18(10), 594-603.

53. Catalan, J., et al. (2017). What influences quality of life in older people living with HIV? AIDS Research and Therapy, 14(1), 22.

54. Erlandson, K. M., et al. (2014). Functional impairment, disability, and frailty in adults aging with HIV-infection. Current HIV/AIDS Reports, 11(3), 279-290.

55. Mafirakureva, N., et al. (2016). Health-related quality of life in HIV/AIDS patients on antiretroviral therapy at a tertiary care facility in Zimbabwe. AIDS Care, 28(7), 904-912.

56. Khan, A. A., Khan, A., \& Bokhari, A. (2010). The HIV epidemic in Pakistan. The Journal of the Pakistan Medical Association, 60(4), 300-307.

57. Pereira, M., \& Canavarro, M. C. (2011). Gender and age differences in quality of life and the impact of psychopathological symptoms among HIV-infected patients. AIDS and Behavior, 15(8), 1857-1869.

58. Mekuria, L. A., et al. (2016). High level of virological suppression among HIV-infected adults receiving combination antiretroviral therapy in Addis Ababa, Ethiopia. Antiviral Therapy, 21(5), 385-396.

59. Sarna, L., et al. (1999). Quality of life in women with symptomatic HIV/AIDS. Journal of Advanced Nursing, 30(3), 597-605.

60. Tran, B. X., et al. (2018). Quality of life improvement, social stigma and antiretroviral treatment adherence: Implications for long-term HIV/AIDS care. AIDS Care, 30(12), 1524-1531.
61. Hikasa, S., et al. (2017). Quality of life of people living with HIV compared with that of the general population in Japan. Journal of Infection and Chemotherapy, 23(10), 698-702.

62. Degroote, S., Vogelaers, D., \& Vandijck, D. M. (2014). What determines health-related quality of life among people living with HIV: An updated review of the literature. Archives of Public Health, 72(1), 40.

63. Rueda, S., et al. (2011). Employment status is associated with both physical and mental health quality of life in people living with HIV. AIDS Care, 23(4), 435-443.

64. Sabino, T. E., et al. (2020). Adherence to antiretroviral treatment and quality of life among transgender women living with HIV/ AIDS in São Paulo, Brazil. AIDS Care, 33(1), 1-8.

65. Gupta, R. K., et al. (2018). HIV-1 drug resistance before initiation or re-initiation of first-line antiretroviral therapy in lowincome and middle-income countries: A systematic review and meta-regression analysis. The Lancet Infectious Diseases, 18(3), 346-355.

66. Chatterjee, S., et al. (2016). Quality of life of HIV/AIDS patients: The influence of CD4 count on It. Natl J Community Med, 7 , 859-863.

67. Gill, C. J., et al. (2002). Relationship of HIV viral loads, CD4 counts, and HAART use to health-related quality of life. Journal of Acquired Immune Deficiency Syndromes, 30(5), 485-492.

68. Protopopescu, C., et al. (2007). Health-related quality of life in HIV-1-infected patients on HAART: A five-years longitudinal analysis accounting for dropout in the APROCO-COPILOTE cohort (ANRS CO-8). Quality of Life Research, 16(4), 577.

69. Armon, C., \& Lichtenstein, K. (2012). The associations among coping, nadir CD4+ T-cell count, and non-HIV-related variables with health-related quality of life among an ambulatory HIV-positive patient population. Quality of Life Research, 21(6), 993-1003.

70. Schroecksnadel, K., et al. (2008). Quality of life and immune activation in patients with HIV-infection. Brain, Behavior, and Immunity, 22(6), 881-889.

71. Bezabhe, W. M., et al. (2016). Adherence to antiretroviral therapy and virologic failure: A meta-analysis. Medicine, 95(15), e3361.

72. The Lancet, H. (2017). $\mathrm{U}=\mathrm{U}$ taking off in 2017. The Lancet HIV, $4(11)$, e475.

Publisher's Note Springer Nature remains neutral with regard to jurisdictional claims in published maps and institutional affiliations.

\section{Authors and Affiliations}

\section{Ali Ahmed ${ }^{1} \cdot$ Muhammad Saqlain $^{2} \cdot$ Naila Bashir $^{3} \cdot$ Juman Dujaili $^{1} \cdot$ Furqan Hashmi $^{4} \cdot$ Faizan Mazhar $^{5} \cdot$ Amjad Khan $^{6}$. Musarat Jabeen ${ }^{7} \cdot$ Ali Blebil $^{1} \cdot$ Ahmed Awaisu $^{8}$ (I)}

Ali Ahmed

ali.ahmed@monash.edu

Muhammad Saqlain

saqlain445@gmail.com

Naila Bashir

drnailapk@gmail.com

Juman Dujaili

Juman.Dujaili@monash.edu
Furqan Hashmi

furqan.pharmacy@pu.edu.pk

Faizan Mazhar

faizan.mazhar@unimi.it

Amjad Khan

amjadkhan@qau.edu.pk

Ali Blebil

ali.blebil@monash.edu 
1 School of Pharmacy, Monash University, Jalan Lagoon Selatan, Bandar Sunway, 47500 Subang Jaya, Selangor, Malaysia

2 Department of Pharmacy, Quaid I Azam University Islamabad, Islamabad, Pakistan

3 HIV Treatment Center, Pims, National AIDs Control Programme, Islamabad, Pakistan

4 University College of Pharmacy, University of the Punjab, Allama Iqbal Campus, Lahore 54000, Pakistan
5 Unit of Clinical Pharmacology, Department of Biomedical and Clinical Sciences L. Sacco, "Luigi Sacco" University Hospital, Università Di Milano, 20157 Milan, Italy

6 Department of Pharmacy, Quaid-I-Azam university, Islamabad, Pakistan

7 HIV Centre PIMS Hospital, Islamabad, Pakistan

8 Department of Clinical Pharmacy \& Practice, College of Pharmacy, QU Health, Qatar University, P.O. Box 2713, Doha, Qatar 